\title{
Simulation-based Analysis of Dispatching Methods on Seaport Container Terminals
}

\author{
Anne Schwientek ${ }^{1^{*}}$, Ann-Kathrin Lange ${ }^{1}$, Carlos Jahn ${ }^{1}$ \\ ${ }^{1}$ Institute of Maritime Logistics, Hamburg University of Technology, Am Schwarzenberg-Campus 4, 21073 Hamburg, \\ Germany; *a.schwientek@tuhh.de
}

\begin{abstract}
Horizontal transport on a container terminal is the interface between quayside and yard. Efficient transport operations between these two areas are an important issue for terminal operators to achieve a high terminal performance. Thereby, an essential task is to dispatch the respective vehicles to pending container transport orders. Despite the large amount of literature in this field, there is no systematic approach investigating the effects of terminal parameters on the performance of dispatching methods. This discrete event simulation study aims to close that research gap for the case of different dynamic dispatching methods. It shows that the performance of a dispatching method depends highly on the terminal's objectives (e.g. maximizing crane productivity, minimizing driven distances). In addition, it reveals that some terminal parameters influence the performance ranking of the dispatching methods. This implies that there is no dominant dispatching method; the choice should rather depend on the specific terminal objectives and parameters.
\end{abstract}

\section{Introduction}

Seaborne containerized trade increases constantly (except in 2009). In 2018, 152 million TEU (Twenty-foot Equivalent Unit) were shipped around the world in contrast to 110 million TEU in 2010 [1]. Within the maritime supply chain, container terminals are interfaces between sea transport and the hinterland, where the handling operations between modes take place and goods are stored. Therefore, they have a high importance for the overall success of maritime transports. The increasing container volumes demand a constant improvement of the terminal parameters, especially of storage space and handling processes.

Besides the increasing cargo volumes, there is another challenging trend for container terminals: vessel sizes grow without cease. Larger vessels make higher demands on ports and terminals in various kinds [2]. On the one hand, physical enlargements of the existing superstructure are necessary to suffice the new vessel dimensions. The port basin and access need to show enough water depth for the vessels' draught and enough width to enable vessels to turn or to pass each other. The terminals' ship-to-shore cranes (STS) need a sufficiently large boom. On the other hand, the handling processes on the terminal need to be updated to fit the changed requirements. As the container carriers mainly expect similar handling times for the Ultra Large Container Ships as for the smaller ones, the terminals need to ensure fast operations at the quayside to stay competitive. Therefore, the terminal equipment has to be able to move a large amount of discharged containers fast enough from the quayside to the yard (or equivalently from the yard to the quayside) and the storage area has to be able to cope with high peak situations. Furthermore, the strategies in the yard have to be optimized to ensure short container handling times with the minimal amount of container rehandling. These challenges lead to the necessity to optimise terminal operations regarding speed and efficiency.

\section{Container Terminal Operations}

To organize container handling processes, container terminals comprise different main functional areas [3, 4]: Quayside, horizontal transport, storage area and landside.

The quayside includes vessels, berths and quay wall. Processes at the quayside are mainly vessel loading and discharging. The term horizontal transport refers to the transport operations between quayside and storage area. In the storage area, either empty or full containers are stored in blocks for a short time period until they are loaded on a truck, train or vessel. The landside consists of the gate, where external trucks enter the terminal area, and the truck and train loading area together with the corresponding processes. In each of these areas, different types of equipment can be used, depending especially on the size, the location and the required productivity of the terminal.

On the quayside, the typical equipment type are STS [5]. Some terminals employ mobile harbour cranes (MHC). While STS productivity ranges usually between 
25 and 35 moves/hour, MHC productivity is only between 15 and 20 moves/hour [3].

Horizontal transport equipment is differentiated between active and passive equipment [4]. Active equipment such as Straddle Carriers (SC) is able to lift a container independently. In contrast, passive equipment like Tractor-Trailer-Units (TT) or Automated Guided Vehicles (AGV) needs another equipment type (e.g. a gantry crane or a STS) to be loaded or unloaded. This implies that more equipment is needed to achieve the same STS productivity if passive equipment is used compared to active equipment as the next vehicle should be always available for the STS to avoid waiting times of the most expensive equipment type [6].

The equipment type used in the storage area depends on various factors such as e.g. the terminal volumes or the required yard capacity. Smaller terminals usually employ Reach Stackers (RS) as they are flexible and have low investment costs. SC are typically employed in medium-sized terminals. They are flexible and productive, but they usually allow only a maximum stacking height of three containers leading to a comparatively high demand for ground space. Other typical equipment types are Rubber-Tyred Gantry Cranes (RTG) and RailMounted Gantry Cranes (RMG). RMG are more productive, have larger span widths and higher stacking heights. Contrarily, RTG are less expensive and more flexible as they are not rail-mounted and, therefore, can change yard blocks whenever necessary [3].

Regarding the landside, external trucks often enter the terminal and bring or collect one or two container(s) directly to/from the storage area. Therefore, for truck (un)loading, the respective storage equipment is used. For train (un)loading, RMG, RS, or SC are employed.

Depending on the employed equipment, the terminal system is defined. Thereby, the terminal system is understood as the combination of technical equipment used for container handling operations [7]. The most common terminal systems are the RTG/TT-system (more than $50 \%$ worldwide) and the SC-system (20-25 \%) [8]. Therefore, these two systems are chosen for the following investigation.

\section{Dispatching in Horizontal Transport}

As discussed above, the term horizontal transport refers to the interface between quayside and yard as one of the main functional areas of a container terminal. It is an essential process to allow for an efficient service to vessels and, therefore, enable fast and reliable quayside operations. Regarding horizontal transport, one main decision problems is the dispatching problem.

Dispatching aims to find an efficient assignment (and sometimes sequence) of transport orders and vehicles (e.g. TT or SC) for a defined time to fulfil the given objective. Typical objectives are to serve the STS continuously, to minimize the driven distances of vehicles or to reduce the amount of used vehicles. Thereby, dynamic and static dispatching can be distinguished [9]. The dynamic approach triggers the assignment at certain events, e.g. if a transport order is completed and a vehicle is available or at the beginning of a shift. This approach is very flexible but also myopic. On the contrary, the static approach (often also called scheduling) generates a longterm plan based on estimates for arrival and operation times. The plan can be optimized in advance using mathematical methods, but is normally not altered during its execution. However, it is highly dependent on the quality of the time estimates [10]. There are also hybrid forms using a static approach with a short rolling horizon. Container terminal processes are quite stochastic. For example, the terminal workload fluctuates depending on the arrival rates of vessels, trucks and trains. Furthermore, important equipment can fail. Therefore, this study focuses on dynamic dispatching methods.

There are several literature reviews on dispatching and scheduling on container terminals. Kizilay and Eliiyi [11] provide a recent overview on quay, yard and integrated scheduling problems. Huang et al. [12] focus on resource allocation problems on container terminals. A detailed analysis of the dispatching literature on container terminals is provided in Schwientek et al. [13].

The respective publications usually develop an own dispatching method (e.g. [14-16]) or compare a few methods for a specific terminal (e.g. [17-20]). Zeng et al. [21] and Liu and Ioannou [19] show that the number of available vehicles influences the dispatching method performance ranking. Garro et al. [22] discuss that dispatching methods developed for AGV are not suitable for SC due to different processes. Schwientek et al. [23] investigate five terminal parameters and three dispatching methods.

The interrelations between terminal parameters and dispatching method performance are so far not investigated systematically. Thus, it is possible that a dispatching method chosen under certain terminal conditions is 
no longer the best solution if the terminal conditions change due to the rather unpredictable environment. Therefore, the research question is, whether certain terminal parameters affect the performance of a dispatching method and if yes, which ones are most important parameters. A simulation study is conducted to approach that question.

\section{Simulation Study}

The simulation study bases on a reference terminal that is implemented in Tecnomatix Plant Simulation using modified data of a real terminal. Both terminal parameters and dispatching methods are varied to investigate the effects of the respective combinations on the efficiency of the horizontal transport.

The reference container terminal reflects a typical RTG/TT-terminal. It focuses on the horizontal transport area framed by the quayside and the yard area. The simulated terminal has a capacity of 1,600,000 TEU, the quay length is $800 \mathrm{~m}$. Eight STS are installed. The yard comprises 20 blocks (6x28 TEU) parallel to the quay wall. For every yard block, there is a RTG available. There are 40 TT (five per STS) on the terminal. Three different vessel types arrive at the terminal: Feeder vessel and two types of deep-sea vessels. Feeder vessels discharge and load on average 500 containers, smaller deepsea vessels discharge and load on average 1,250 containers and larger deep-sea vessels discharge and load on average 3,500 containers.

The choice of dispatching methods, terminal parameters and terminal objectives bases on a preceding literature analysis [13]. The terminal objectives typically relate to an object such as a vessel, STS, or vehicle. Examples for objectives are: to minimize the makespan of a vessel, to minimize the departure delay, to maximize STS productivity or to minimize STS wait time, to maximize vehicle productivity or to minimize vehicle wait time, travel distance or fleet size. The majority of dispatching studies focuses on vessel- or vehicle-related objectives [13]. Thus, output parameters of this study are exemplarily STS productivity and distances driven by the vehicles.

\subsection{Investigated Dispatching Methods}

Five different dynamic dispatching methods are investigated: fixed assignment of vehicles to a STS, distancebased, inventory-based, time-based, and a hybrid method. The chosen dispatching methods showed a good performance in comparison to others in earlier studies as described in the following.

The fixed method is the most used method in practice. Thereby, a fixed number of vehicles is assigned to a particular STS serving only this specific STS. This is a very simple method which is easy to implement and comfortable to the terminal personnel. However, the fixed method is highly inefficient as roughly half of the trips between quay and yard are empty trips (assuming singlecycle operation of the STS, i.e. discharging and loading are separate processes).

Studies by Kim and Bae [24], Koster et al. [20], and Meer [25] recommend a distance-based dispatching method. The implemented distance-based method aims to minimize the driven distances of the vehicle by always assigning the transport order with the nearest starting location.

The inventory-based method is a dispatching method referring to Briskorn et al. [16]. It was evaluated positively in several other publications. Thereby, an available vehicle is assigned to a transport order that belongs to the STS with the smallest inventory. The inventory of a STS is understood as the number of transport orders that is already assigned to this specific STS.

Cao et al. [26] state that a time-based method performs higher than a genetic algorithm. The implemented time-based dispatching method chooses the transport order for a vehicle that waits longest for assignment. It focuses like the inventory-based method on the STS as the waiting time of a transport order potentially leads to waiting time for the STS.

A hybrid dispatching method is positively evaluated by Meer [25], Angeloudis and Bell [27] and Song and Huang [28]. The implemented hybrid method combines the distance-based, the inventory-based and the timebased method. It calculates a score that comprises separate scores for shortest distance, lowest inventory and longest waiting time.

\subsection{Varied Terminal Parameters}

As with the dispatching methods, terminal parameters are chosen that were identified to influence the dispatching method performance ranking or that appear to be possibly relevant. Analyzing the parameters being varied in the sensitivity analyses of relevant studies, the most considered parameter is the number of vehicles, followed by the number of STS and yard cranes. The number of STS and yard cranes is typically motivated by modifying the terminal size. Furthermore, problem parameters are varied 
like the time between jobs, time between vessels, STS load/discharge combination or others. In addition, layout-related parameters are of interest such as distance between quay and yard, number of blocks or the stacking height in the yard. Scarcely investigated parameters are the degree of stochasticity, vehicle capacity or vehicle speed [13]. Eleven terminal parameters are evaluated in this study regarding their influence on the performance of dispatching methods:

1. Number of vehicles

2. Speed of vehicles

3. Yard block assignment to containers

4. Utilization of seaside capacity

5. Vessel sizes

6. Terminal size

7. Equipment type

8. Range of handling times

9. STS handling rate

10. Quay layout

11. Share of landside traffic

The number of vehicles on the terminal for the different simulation runs ranges between 24 and 48 . It reflects values of 3, 4, 5 or 6 TT per STS. The number of vehicles is analyzed in many respective sensitivity analyses. Zeng et al. [21] and Liu and Ioannou [19] indicate that this parameter influences the performance ranking of dispatching methods. The speed of vehicles is rarely investigated. Behera et al. [29] find a certain influence of TT speed on handling volumes. The varied values are $5.6 \mathrm{~m} / \mathrm{s}, 8.4 \mathrm{~m} / \mathrm{s}$, and $11 \mathrm{~m} / \mathrm{s}$ for TT.

The assignment of containers to yard blocks influences the dispatching substantially as it determines the start respectively the destination of the transport orders. Within the simulation study, the investigated values are random, three blocks close to the respective STS, three defined blocks distributed over the yard area and mixed, which is a combination of close for export containers and three for import containers.

The ulitization of the seaside capacity is an essential container terminal parameter. A high capacity utilization leads to fewer options to reduce waiting times and distances [25]. This might influence the dispatching method ranking. Varied values are $50 \%, 63 \%, 75 \%, 88 \%$, and $100 \%$. The size of vessels arriving at the terminal affects the structure of the workload for the horizontal transport. This directly affects the direction of the transport orders and therefore the dispatching. Varied values are only small vessels (average 500 containers), typical size distribution, and only large vessels (average 1250 and 3500 containers). The terminal size refers to the maximum container handling capacity of the quayside. It influences the distances the vehicles have to drive on the terminal. Investigated values are $0.8,1.2,1.6$, and 3.2 million TEU/a. These numbers are representative for typical container terminals.

As described earlier, different equipment types are employed for the horizontal transport on container terminals. The most common types are TT and SC. While TT represent passive equipment, SC are active equipment. This influences the container handling processes. TT and STS respectively RTG have to wait for each other to transfer a container. SC handle a container independently from the cranes. This fact might also affect the dispatching results. Garro et al. [22] state that a dispatching method developed for AGV is not appropriate for SC.

The range of handling times is a value for the simulation model. The handling times are assumed to follow a triangular distribution. The range reflects the difference between the minimum and the maximum of the cranes' handling times. Kim and Bae [24] and Meersmans and Wagelmans [30] state that the range of handling times does not affect the ranking of dispatching methods. The varied values are deterministic handling times, normal, and large.

The average handling rate of STS are also investigated. The handling speed depends on several factors as e.g. the crane driver capabilities, the wind conditions, or the vessel type. Varied values are 24, 30, and 36 moves/hour.

The quay layout of a container terminal is often just straight. However, depending on the terminal location, many other layouts are possible. For the simulation study, besides the straight layout two other layouts are chosen: L-shaped and rectangular (see Figure 1).

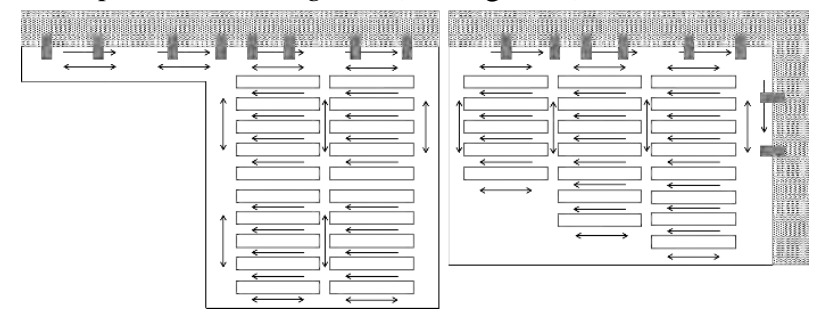

Figure 1: Alternative Quay Layouts (left L-shaped, right rectangular)

On a typical RTG/TT-terminal, external trucks enter the terminal area and drive directly into the yard area for loading respectively unloading. This landside process disturbs the RTG handling process and it might delay 
horizontal transport operations. To investigate the influence of the landside traffic, typical variations of import/export containers are assumed (values: $0 \%$, $15 \%$, $30 \%, 45 \%)$.

\subsection{Design of Experiment}

To determine the influence of each terminal characteristic, a reference terminal is defined based on the terminal specifications analyzed before. The reference terminal uses TT for horizontal transport. $40 \mathrm{TT}$ are available driving with a speed of $8.4 \mathrm{~m} / \mathrm{s}$. The yard block assignment of containers is random and the utilization of the seaside capacity is $75 \%$. Vessels of all sizes arrive at the terminal. The terminal size is 1.6 million TEU/a. The range of handling times is normal, the handling times of STS is 30 moves/hour. The quay layout is straight; the share of landside traffic is $0 \%$.

Based on this reference terminal, every terminal characteristic is modified ceteris paribus to analyze the effects of a parameter variation. Each parameter variation is simulated in combination with the five different dispatching methods. This leads to 29 parameter variations and combined with five different dispatching methods to a total of 145 experiments. For each experiment, 50 simulation runs are conducted.

\section{Simulation Results}

The fixed assignment of TT to a STS is - both in terms of STS productivity and distances driven- the worst dispatching method. The time-based method leads on average to the highest STS productivity. It is $4 \%$ higher compared to the STS productivity of the fixed method. The inventory-based and the hybrid method perform similarly to the time-based method. The second lowest STS productivity is achieved with the distance-based method.

Regarding the driven distances per container, the distance-based method performs best. It reduces the mean distance by $8 \%$ compared to the fixed method. The hybrid method performs similar. Thus, the hybrid method leads to a high STS productivity as well as to low driven distances per container. The time- and the inventorybased method lead to similar results as the fixed method.

These results are plausible as the distance-based method focuses on minimizing the driven distances of the TT, and the inventory- and the time-based method aim to avoid waiting times for the STS. The hybrid method combines the strengths of all three methods.
Two of the eleven evaluated terminal parameters influence the performance ranking of the dispatching methods, namely the yard block assignment to containers and the equipment type. This means that if the value of one of these parameters changes, it would be beneficial to apply a different dispatching method. The variation of all other evaluated terminal parameters led only to minor or no changes of the performance ranking of the dispatching methods. Their impact on the STS performance and driven distances per container are explained afterwards.

\subsection{Effects of Yard Block Assignment to Containers}

As Figure 2 shows, the assignment of containers to yard blocks affects the dispatching method performance ranking regarding the terminal objective to minimize the driven distances per container. If the assignment is random or the containers are transported to three defined blocks distributed over the yard area, the distance-based and the hybrid method are the best choices. If the containers are assigned to yard block close to the STS, the fixed method (which is usually the least successful one) performs best.

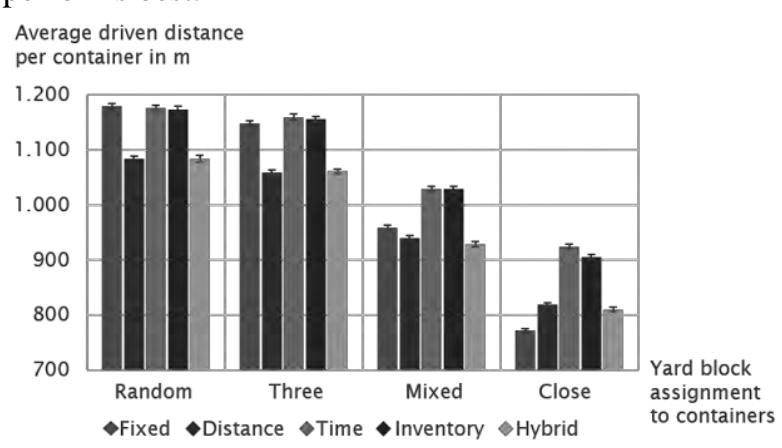

Figure 2: Average Driven Distances per Container Depending on the Yard Block Assignment

An explanation might be the short driving distances for the TT between quayside and yard. Using the fixed method, the TT always have short driving distances as the containers are located very close. All other methods allow the TT to drive a longer distance to pick up or bring a container from / to a yard block further away when no other transport order is available. However, this yard block assignment might lead to longer driving distances and possible congestion for external trucks on the container terminal, as they are required to bring and pick up the containers near the quayside.

The STS productivity is highest in case of a random assignment of containers to yard blocks (see Figure 3). This is due to the fact, that the TT approach more yard 
blocks and the risk to wait for a busy RTG is lower. If the TT approach only three yard blocks, the probability is higher that they have to wait for the RTG and let the STS wait. The time-, inventory-based and hybrid method are similarly suited for all three assignments. The second lowest STS productivity is achieved with the distancebased method and the lowest with the fixed method.

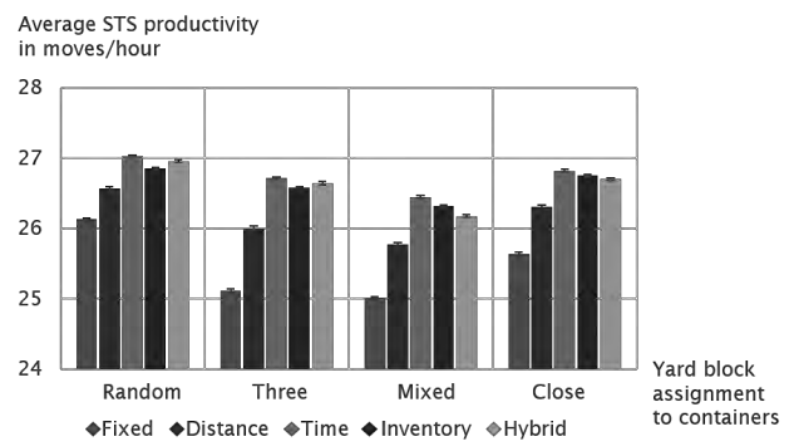

Figure 3: Average STS Productivity Depending on the Yard Block Assignment

\subsection{Effects of Equipment Type}

The chosen type of equipment affects the dispatching method performance ranking regarding the driven distances per container (see Figure 4). If TT are employed, the distance-based and the hybrid method perform both well, reducing distances by approximately $9 \%$ compared to the fixed method. The time- and the inventory-based method are on a similar level as the fixed method. If SC are used for horizontal transport and yard operations, the fixed assignment of SC to STS leads to the shortest driven distances per container. The hybrid method is the next best followed by the distance-, inventory- and timebased method.

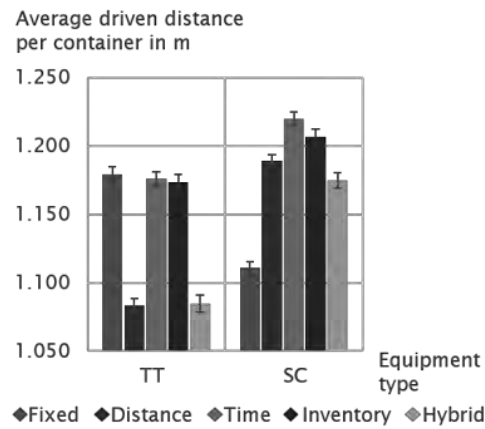

Figure 4: Average Driven Distances per Container Depending on the Equipment Type

The STS productivity is equally high for all evaluated dispatching methods for the SC case and it is higher than for the TT case (see Figure 5). For TT, the time-based methods generates the best results followed shortly by all other methods.

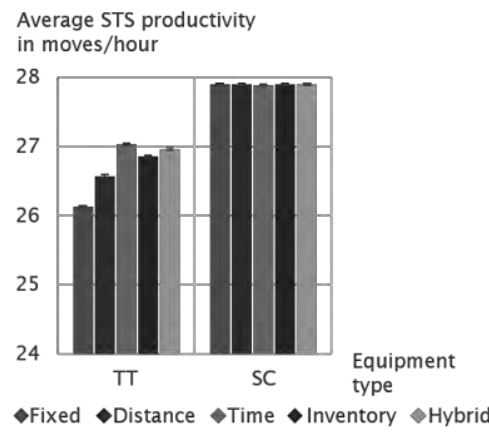

Figure 5: Average STS Productivity Depending on the Equipment Type

\subsection{Effects of Other Terminal Parameters}

All other evaluated terminal parameters do not affect the dispatching method ranking, i.e. one dispatching method is always the best choice to achieve a certain terminal objective when the respective terminal parameter is varied. However, terminal parameters do often affect the STS productivity and the driven distances of the vehicles. Therefore, a short description of their impact is given hereafter.

The number of vehicles used for horizontal transport influences both STS productivity and driven distances. If the number is too low, the STS productivity drops significantly below 24 moves/hour for the time-, inventorybased and hybrid method and down to 19 moves/hour for the fixed assignment method. This means that there are many delays in horizontal transport operations and waiting times for the STS. If 48 TT are available, all dispatching methods lead to a similarly high STS productivity. However, this also implies longer distances for the distance-based and the hybrid method. This is caused by the fact that on average, there are less transport orders to choose from for a TT and, therefore, the potential to reduce distances is lower.

A variation of the vehicle speed does not affect the driven distances. Likewise, an increase from $8.4 \mathrm{~m} / \mathrm{s}$ to $11 \mathrm{~m} / \mathrm{s}$ does not improve the STS productivity. Only a decrease to $5.6 \mathrm{~m} / \mathrm{s}$ reduces the STS productivity slightly.

A variation of the utilization of the seaside capacity does not affect the dispatching method performance ranking. If the capacity usage decreases, the STS productivity improves slightly because there is the same amount of vehicles available for less transport orders leading to reduced waiting times for a transport order to be executed. At the same time, the driven distances rise because a free vehicle finds a smaller number of transport orders 
to choose from reducing the possibilities to minimize distances.

Changing the vessel size and thereby the structure of the workload for the horizontal transport influences the STS productivity. If the workload structure is relatively balanced, the STS productivity increases by approximately 0.5 moves/hour.

The terminal size correlates with the driven distances. Interestingly, the gap between the dispatching methods is relatively small for smaller terminals (4\%), and increases with the terminal size. For the largest terminal in this study, the gap between distance-based and hybrid on the one hand and inventory-, time-based and fixed assignment is approximately $18 \%$. This implies that the choice of a dispatching method is more important for larger container terminals than for smaller terminals. Regarding STS productivity, the fixed assignment method is almost as good as the other dispatching methods for the case of the small terminal.

The range of handling times has no impact on the driven distances. However, a large range reduces the STS productivity by more than 1 move/hour compared to a normal range. This is plausible as a high range of handling times especially in the yard increases the risk that a TT arrives late at the STS causing waiting times for the STS.

Shorter handling rates of the STS reduce the STS productivity as expected. If the number of available TT stays constant while the handling times improve, the beneficial effect on the STS productivity lessens. A variation of the handling times does not affect the driven distances.

A variation of the quay layout influences the STS productivity not significantly. Only for the case of the Lshaped layout, the STS productivity decreases slightly. However, the quay layout affects the driven distances. The rectangular layout leads to distances between 1000 and $1100 \mathrm{~m}$ (depending on the dispatching method), the straight layout to distances between 1080 and $1180 \mathrm{~m}$ and the L-shaped layout leads to distances of more than $1300 \mathrm{~m}$.

The landside traffic affects the STS productivity negatively if the percentage rises above $30 \%$. This means that there are more occasions when a RTG serves an external truck while an urgent order for an internal transport should be handled. There is no influence of the landside traffic on the driven distances.

\section{Conclusion}

This simulation study shows a first start to evaluate the interrelations between container terminal parameters and dispatching method performance depending on different terminal objectives. Especially the choice of the terminal objectives influences the choice of the dispatching method. Five different dispatching methods are examined on their effect on STS productivity and driven distance per container of horizontal transport vehicle. These methods are the fixed assignment of vehicles to STS, a distance-, a time-, an inventory-based and a hybrid method. Two terminal parameters out of eleven have been found out to have an impact on the performance ranking of dispatching methods and, therefore, on the choice of the best dispatching method for a specific terminal to achieve a specific objective. These parameters are the assignment of containers to yards block and the equipment type used for the horizontal transport. All other evaluated terminals parameters are analyzed on their impact on STS productivity and on the driven distance per container.

Future research aims to systematically extend this study by further parameters, dispatching methods and terminal objectives. Especially the hybrid method performed well for both analyzed performance indicators. It combines the benefits of the distance-, time- and inventory-based methods while still being easy to implement. In future research, different weightings of the scores of the three singular methods can be evaluated to highlight potential for further improvement. Furthermore, it could be reasonable to change the weightings of the hybrid dispatching method during operation. For example, if a large deep-sea vessel is moored at the quay side or if less vehicles are available, the focus should be on STS productivity, while in case of a lower utilization of the seaside capacity, a focus on reducing the driven distances would be beneficial.

Furthermore, future research may focus on the evaluation of using meta-heuristics, e.g. genetic algorithms, for the dispatching process. Here, especially the computational times and the accuracy are important to ensure a sufficient transferability to real life container terminals. The use of a rolling horizon might prove a possible solution. Another promising field of interest is the integration of landside processes, e.g. the implementation of a truck appointment system, and their impact on the dispatching strategies in the horizontal transport, especially for terminals with a high share of import and export containers. 


\section{References}

[1] UNCTAD. Review of Maritime Transport 2019. New York, Geneva, United Nations, 2019.

[2] Gharehgozli AH, Roy D, Koster RBM de. Sea container terminals: New technologies and OR models. Marit Econ Logist 2016;18:103-140.

[3] Brinkmann B. Seehäfen - Planung und Entwurf. Berlin, Springer, 2005.

[4] Steenken D, Voß S, Stahlbock R. Container terminal operation and operations research - a classification and literature review. OR Spectrum 2004;26:3-49.

[5] Kemme N. Design and Operation of Automated Container Storage Systems. Heidelberg, PhysicaVerlag HD, 2013.

[6] Bae HY, Choe R, Park T, Ryu KR. Comparison of operations of AGVs and ALVs in an automated container terminal. J Intell Manuf 2011;22:413426.

[7] Brinkmann B. Operations Systems of Container Terminals: A Compendious Overview. Edited by Böse JW. New York, Springer, 2011, pp. 25-39.

[8] Wiese J, Kliewer N, Suhl L. A Survey of Container Terminal Characteristics and Equipment Types, 2009.

[9] Bian Z, Yang Y, Mi W, Mi C. Dispatching Electric AGVs in Automated Container Terminals with Long Travelling Distance. Journal of Coastal Research 2015;73:75-81.

[10] Grunow M, Günther H-O, Lehmann M. Strategies for dispatching AGVs at automated seaport container terminals. OR Spectrum 2006;28:587-610.

[11] Kizilay D, Eliiyi DT. A comprehensive review of quay crane scheduling, yard operations and integrations thereof in container terminals. Flexible Serv Manuf J 2020.

[12] Huang J, Wang F, Shi N. Resource allocation problems in port operations: A literature review. Edited by Zhu Q, Wang S, Chai J, Yu L, Institute of Electrical and Electronics Engineers Inc, 2014, pp. 154-158.

[13] Schwientek AK, Lange A-K, Jahn C. Literature classification on dispatching of container terminal vehicles. Edited by Jahn C, Kersten W, Ringle CM. Berlin, epubli, 2017, pp. 3-36.

[14] Lee LH, Chew EP, Tan KC, Wang Y. Vehicle dispatching algorithms for container transshipment hubs. OR Spectrum 2010;32:663-685.

[15] Bian Z, Zhang Y, Zhang X, Xiao Y, Chai J, Mi W. Simulation-based AGV dispatching in automated container terminal, Institute of Electrical and Electronics Engineers Inc, 2019, pp. 414-420.

[16] Briskorn D, Drexl A, Hartmann S. Inventorybased dispatching of automated guided vehicles on container terminals. OR Spect. 2006;28:611-630.

[17] Nguyen VD, Kim KH. Heuristic algorithms for constructing transporter pools in container terminals. IEEE Trans. Intell. Transp. Syst. 2013;14:517-526.

[18] Grunow M, Günther H-O, Lehmann M. Dispatching multi-load AGVs in highly automated seaport container terminals. OR Spectrum 2004;26:211235.

[19] Liu C-I, Ioannou PA. A comparison of different AGV dispatching rules in an automated container terminal. Edited by Lee D.-H, Srinivasan D, Cheu R.L, Institute of Electrical and Electronics Engineers Inc, 2002, pp. 880-885.

[20] Koster RBM de, Le-Anh T, Meer JR van der. Testing and classifying vehicle dispatching rules in three real-world settings. Journal of Operations Management 2004;22:369-386.

[21] Zeng Q, Yang Z, Lai L. Models and algorithms for multi-crane oriented scheduling method in container terminals. Transp. Policy 2009;16:271-278.

[22] Garro A, Monaco MF, Russo W, Sammarra M, Sorrentino G. Agent-based simulation for the evaluation of a new dispatching model for the straddle carrier pooling problem. SIMULATION 2015;91:181-202.

[23] Schwientek AK, Lange A-K, Jahn C. SimulationBased Analysis of Dispatching Methods on Seaport Container Terminals. Edited by Freitag M, Kotzab H, Pannek J, Springer International Publishing, 2018, pp. 167-171.

[24] Kim KH, Bae JW. A Look-Ahead Dispatching Method for Automated Guided Vehicles in Automated Port Container Terminals. Transportation Science 2004;38:224-234.

[25] Meer JR van der. Operational control of internal transport. [Rotterdam], [ERIM], 2000.

[26] Cao J, Shi Q, Lee D-H. A decision support method for truck scheduling and storage allocation problem at container. Tinshhua Sci. Technol. 2008;13:211-216.

[27] Angeloudis P, Bell MGH. An uncertainty-aware AGV assignment algorithm for automated container terminals. Transportation Research Part E: Logistics and Transportation Review 2010;46:354-366.

[28] Song LQ, Huang SY. A hybrid metaheuristic method for dispatching automated guided vehicles in container terminals, 2013, pp. 52-59.

[29] Behera JM, Diamond NT, Bhuta CJ, Thorpe GR. The impact of job assignment rules for straddle carriers on the throughput of container terminals. ATR 2000;34:415-444.

[30] Meersmans PJM, Wagelmans APM. Effective Algorithms for Integrated Scheduling of Handling Equipment at Automated Container Terminals. Rotterdam, 2001. 